\title{
The Impact Exerted on Clinical Outcomes of Patients With Chronic Heart Failure by Aldosterone Receptor Antagonists: A Meta-Analysis of Randomized Controlled Trials
}

\author{
Renato De Vecchis ${ }^{\mathrm{a}, \mathrm{d}}$, Claudio Cantatrione ${ }^{\mathrm{a}}$, Damiana Mazzei ${ }^{\mathrm{a}}$, Augusto Barone ${ }^{\mathrm{b}}$, \\ Nicola Maurea ${ }^{\mathrm{c}}$
}

\begin{abstract}
Background: Aldosterone receptor antagonists (ARAs) have been associated with improved clinical outcomes in patients with heart failure with reduced left ventricular ejection fraction (HFREF), but not in those with heart failure with preserved left ventricular ejection fraction (HFpEF). With the aim to study this topic more deeply, we carried out a meta-analysis of selective and non-selective ARAs in HFREF and HFpEF.
\end{abstract}

Methods: We searched PubMed and Scopus databases. We decided to incorporate in the meta-analysis only randomized controlled trials (RCTs) of ARAs in patients with chronic heart failure (CHF) if they met the following criteria: experimental groups included patients with CHF treated with ARAs in addition to the conventional therapy; control groups included patients with $\mathrm{CHF}$ receiving conventional therapy without ARAs. Outcomes of interest were all-cause death, hospitalizations from cardiovascular cause, hyperkalemia, or gynecomastia.

Results: We detected 15 studies representing 15,671 patients. ARAs were associated with a reduced odds of all-cause death (odds ratio (OR): $0.79 ; 95 \%$ confidence interval (CI): 0.73 - 0.87) and hospitalizations from cardiovascular cause (OR: 0.73 ; 95\% CI: $0.61-0.89$ ). However, subgroup analysis showed that these advantages were limited to HFREF (all-cause death: OR: 0.77, 95\% CI: 0.69 - 0.84; hospitalizations from cardiovascular cause: OR: $0.66,95 \%$ CI: $0.51-0.85$ ), but they did not affect the HFpEF group (all-cause death: OR: 0.91, 95\% CI: 0.76 - 1.1; hospitalizations from cardiovascular cause: OR: $0.85,95 \%$ CI: $0.7-1.09)$. ARAs increased the risk of hyperkalemia (OR: 2.17; 95\% CI: 1.88 - 2.5). Non-selective ARAs, but not selective

Manuscript accepted for publication December 19, 2016

aCardiology Unit, Presidio Sanitario Intermedio "Elena d'Aosta”, ASL Napoli 1 Centro, Napoli, Italy

bDivision of Sports Medicine, Presidio Sanitario Intermedio "Elena d'Aosta", ASL Napoli 1 Centro, Napoli, Italy

'Division of Cardiology, Istituto Nazionale per lo Studio e la Cura dei Tumori, "Fondazione Giovanni Pascale" IRCCS, Napoli, Italy

${ }^{\mathrm{d} C}$ Corresponding Author: Renato De Vecchis, Cardiology Unit, Presidio Sanitario Intermedio "Elena d'Aosta", ASL Napoli 1 Centro, Napoli, Italy (personal postal address: via P.Gaurico 21, 80125 Napoli, Italy).

Email: devecchis.erre@virgilio.it

doi: https://doi.org/10.14740/jocmr2851w
ARAs, increased the risk of gynecomastia (OR: 8.22, 95\% CI: 4.9 13.81 vs. OR: $0.74,95 \%$ CI: $0.43-1.27)$.

Conclusions: ARAs reduced the risk of adverse cardiac events in HFREF but not HFpEF. In particular, ARA use in HFpEF patients is questionable, since in this CHF type, no significant improvement in all-cause death and cardiovascular hospitalizations was demonstrated with ARA treatment, in the face of the well-known risks of hyperkalemia and/or gynecomastia that chronic ARA therapy entails. Selective ARAs were equally effective as non-selective ARAs, without the risk of gynecomastia.

Keywords: Heart failure; Heart failure with reduced ejection fraction; Heart failure with preserved ejection fraction; Aldosterone receptor antagonists; Meta-analysis

\section{Introduction}

The renin-angiotensin-aldosterone system (RAAS) is activated during chronic heart failure (CHF) but this activation implies unfavorable changes of hemodynamics and cardiac work. In short, the pathophysiological mechanisms underlying the overactivation of the RAAS share some elements with the pathophysiologic dynamics involved in the stimulation of antidiuretic hormone release from the posterior pituitary [1]. In fact, a common trigger, as regards the stimulation of the two systems, is represented by the relative condition of underfilling of the arterial vascular compartment with a simultaneous increase in the volume of blood contained in the systemic venous compartment. Underfilling of the arterial circulation due to reduced cardiac output results in unloading of the arterial baroreceptors, which will increase non-osmotic vasopressin release and activate the sympathetic nervous system (SNS) and RAAS. This adrenergic surge promotes additional stimulation of the RAAS.

Aldosterone, produced by the zona glomerulosa of the adrenal cortex, promotes avid reabsorption of sodium and water at the level of the collecting duct in the distal nephron. In CHF, increased volume of extracellular fluids, elicited by aldosterone through the increase in sodium and water reabsorption in the distal nephron, would be aimed at retrieving an effective arterial blood volume, so as to potentiate the cardiac pump 


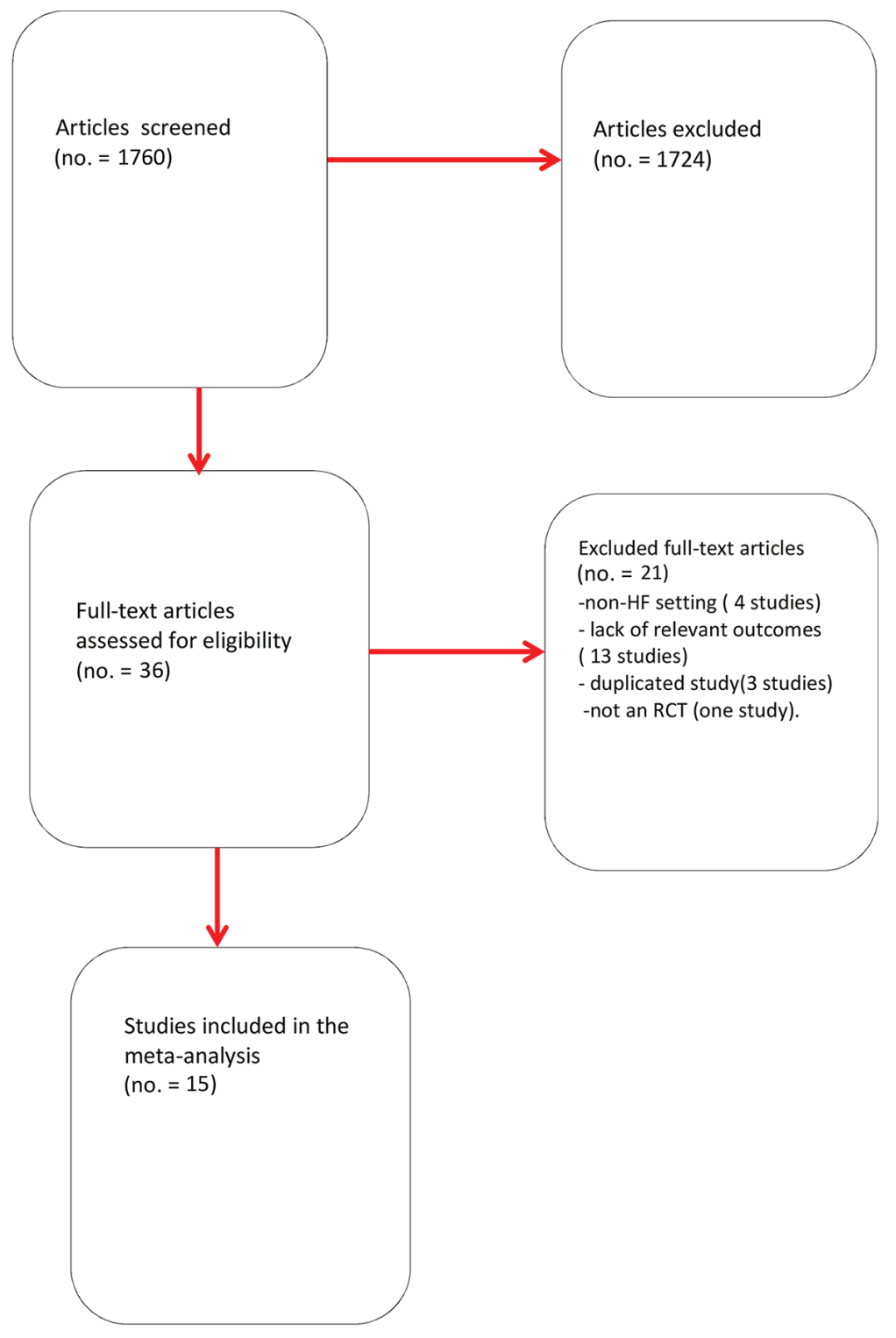

Figure 1. Flow diagram for meta-analysis. Overview of process used to identify studies suitable for inclusion in the meta-analysis.

function through the Frank-Starling mechanism [2-5]. However, retrieval of the pump effectiveness is not really achieved, because increased preload causes a deleterious aggravation of the cardiac work in the decompensated left ventricle, which entails a progressively increasing defaillance of the working myocardial fibers. So in the failing heart, an increase in left ventricular end-diastolic pressure occurs as a result of the increase in circulating fluid volume, induced by aldosterone; however, this does not result in any increase in cardiac output but rather causes a state of hemodynamic congestion [6], initially subclinical, then clinically evident (dyspnea on minimal efforts, peripheral edema, etc.).

Because of RAAS activation, the CHF patient manifests increased ventricular stress, with impaired left ventricular systolic function, and impaired systemic venous drainage, i.e., slowing down of systemic venous flow.
Thus, RAAS overactivation is mirrored by clinical worsening of heart failure with aggravation of dyspnea and edema; more importantly, a reduction of the patient's life expectancy has been proven in the case of RAAS overstimulation. For these reasons, huge efforts have been made to obtain molecules that have the property to efficaciously antagonize RAAS overstimulation. ACE inhibitors, angiotensin receptor blockers (ARBs), and aldosterone receptor antagonists (ARAs) are the three classes of drugs that have been proven to effectively counter the RAAS overstimulation typically found in CHF.

Several studies showed that despite maximal RAAS blockage with ACE inhibitors and/or ARBs, plasma aldosterone levels remain elevated in patients with heart failure [7-9]. Thus, ARAs were introduced in the therapy of CHF, following the demonstration of their protective effect on the failing heart. Indeed, several trials documented improved survival 


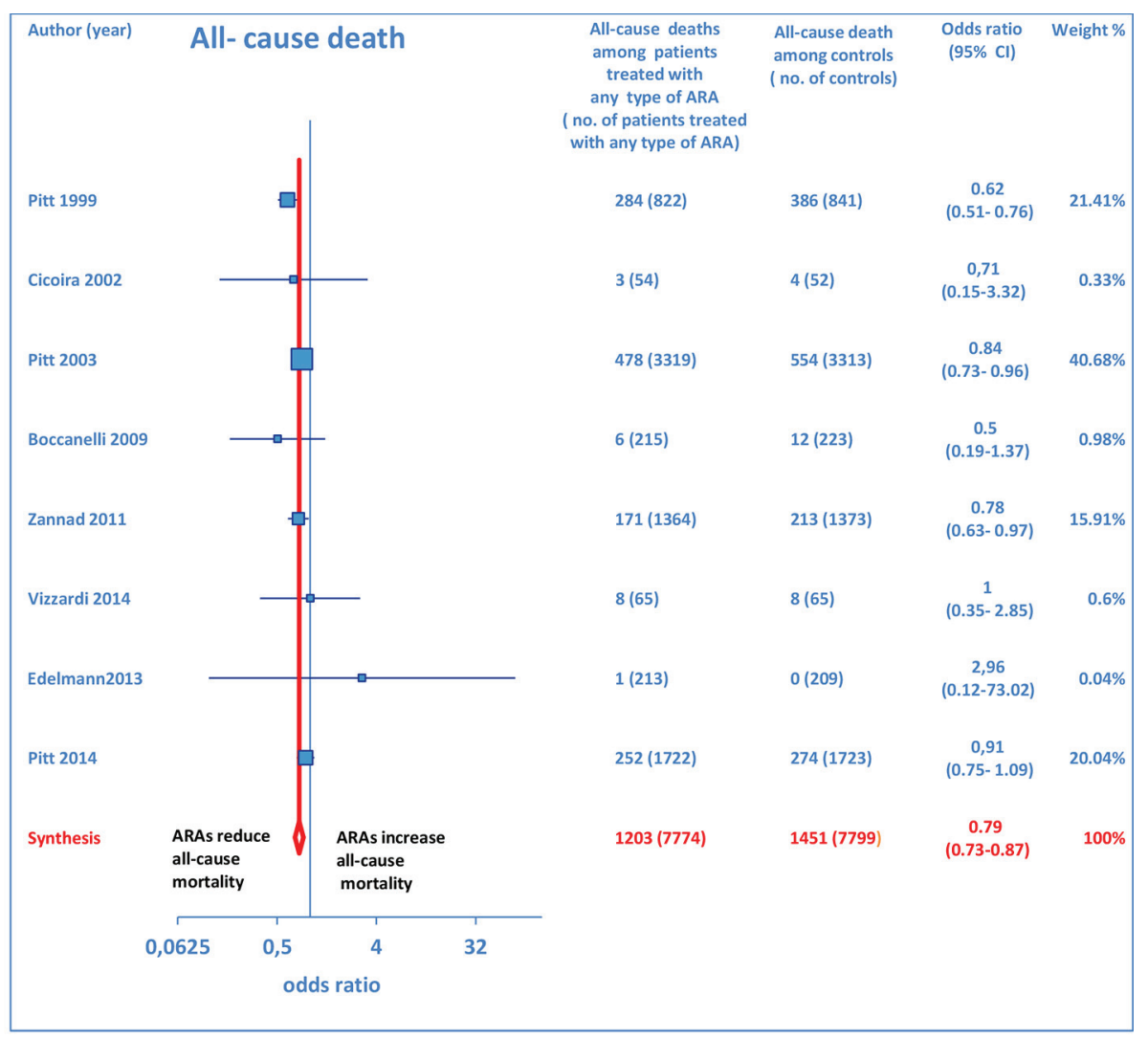

Heterogeneity : $I^{2}=30.67 \% ; Q=10.096, p=0.1831$

Figure 2. Forest plot of all-cause mortality with ARA use in HF. Eight trials reported all-cause mortality rates with ARA use in HF patients compared to controls.

for patients treated with ARAs, compared with controls who received only beta-blockers, diuretics, and ACE inhibitors [10-12]. The aldosterone receptor blockers include both nonselective (spironolactone and canrenone) and selective antagonists (eplerenone and finerenone). Eplerenone was synthesized through chemical modification of spironolactone in order to enhance binding of mineralocorticoid receptors while reducing off-target binding to progesterone or androgen receptors [13]. Eplerenone is associated with lower rates of impotence, gynecomastia, or breast pain in comparison to spironolactone $[11,12]$.

\footnotetext{
Aims

The aim of this meta-analysis of randomized controlled trials (RCTs) was to verify the impact of ARAs on some hard endpoints (all-cause death and hospitalizations from cardiovascular cause), making a comparative evaluation of these outcomes in CHF patients with reduced left ventricular ejection fraction (HFREF) and in those with preserved left ventricular ejection fraction (HFpEF), respectively. The meta-analysis was then extended to the comparison between non-selective and selective ARAs, with regard to their respective effects on the above-mentioned clinical endpoints, and also as regards the
}

respective side effects, such as hyperkalemia or gynecomastia.

\section{Methods}

\section{Eligibility criteria}

In our meta-analysis, we considered exclusively RCTs. These studies were included if they met the following criteria: experimental groups included patients with CHF treated with ARAs in addition to the conventional therapy; control groups included patients with CHF receiving conventional therapy without ARAs. In addition, the studies selected for the meta-analysis only included patients older than 18 years. Animal experimental studies as well as case reports of ARA administration in patients with $\mathrm{CHF}$ were eliminated from the meta-analysis. Similarly, all studies not written in English, duplicated studies, non-randomized studies, review articles, editorials, and expert opinions were excluded.

Outcomes of interest were all-cause mortality, cardiovascular hospitalizations, hyperkalemia, or gynecomastia. The efficacy endpoints, i.e., mortality and hospitalizations, as well as the safety endpoints, namely episodes of hyperkalemia or occurrence of gynecomastia, were evaluated in both patients with 


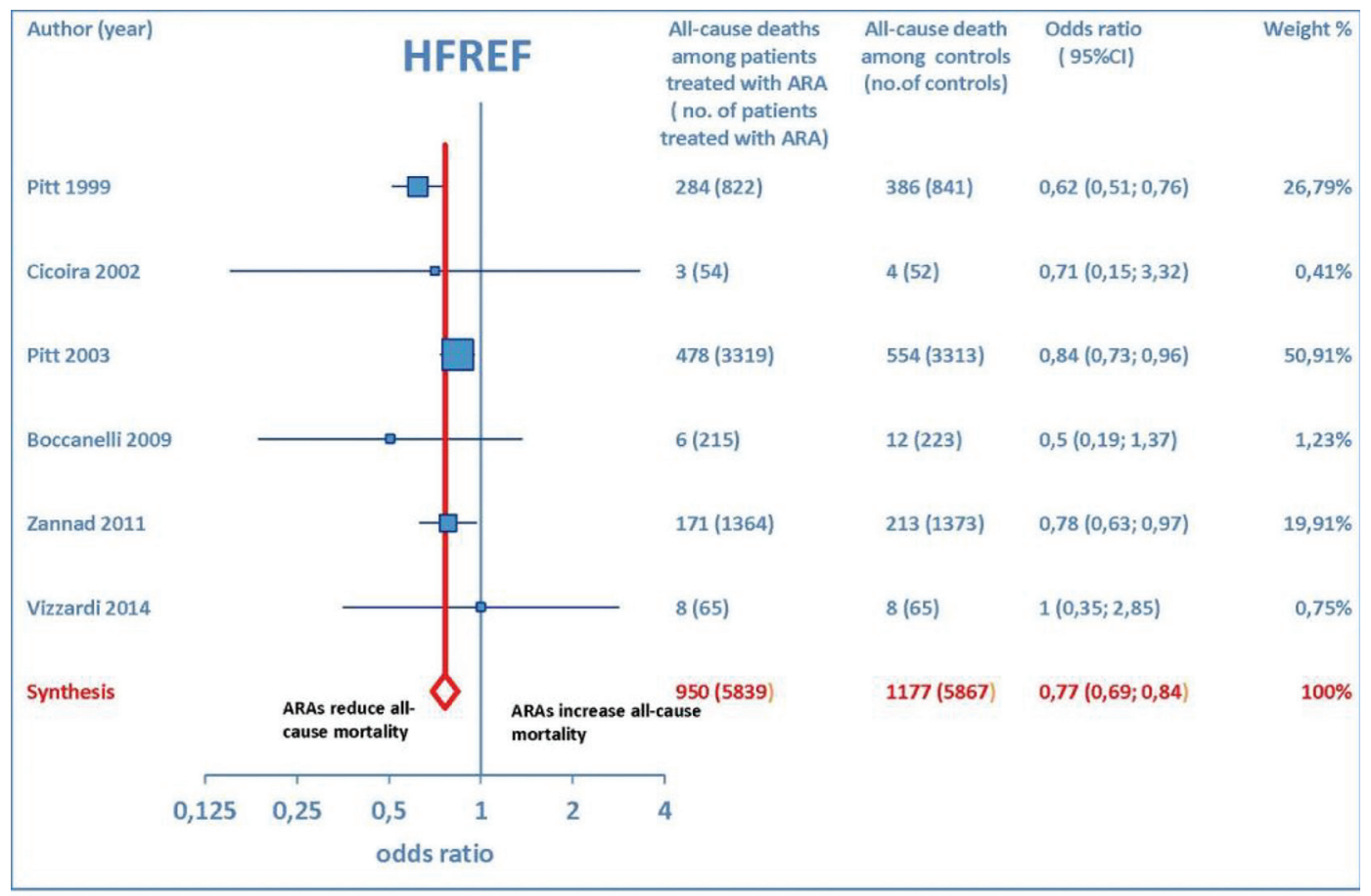

Heterogeneity: $I^{2}=28.10 \% ; Q=6.954, p=0.224$

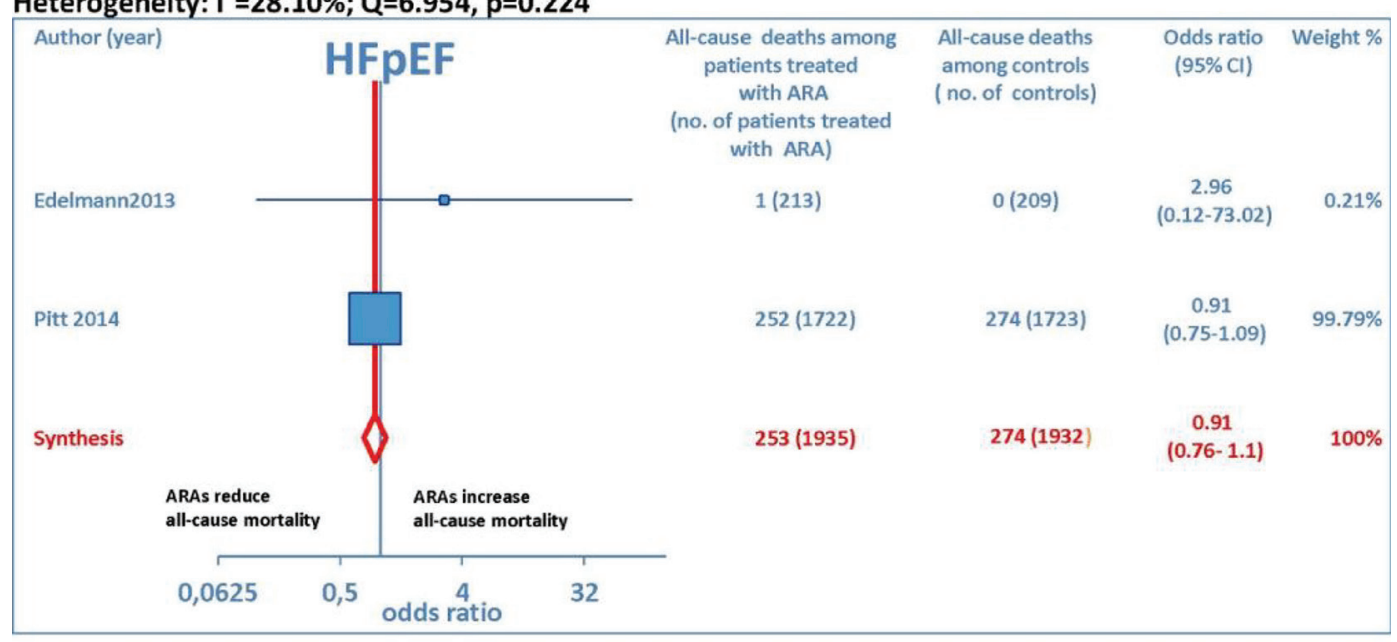

\section{Heterogeneity: $I^{2}=0.00 \% ; Q=0.52, p=0.4705$}

Figure 3. The forest plot of all-cause mortality with ARA use has been subdivided according to HF type.

heart failure with HFREF and those with HFpEF as compared to control groups. Moreover, a comparison was made between non-selective ARAs (e.g., spironolactone or canrenone) and selective ARAs (e.g., eplerenone or finerenone) with regard to efficacy outcomes as well as side effects.

\section{Study selection}

A systematic search using some related terms was conducted using the PubMed and Scopus electronic archives (from June 1995 to June 30,2016 ).
The study was performed according to the guidelines and recommendations expressed in the "Preferred Reporting Items for Systematic reviews and Meta-Analyses (PRISMA)" [14] statement. Search terms included "aldosterone receptor antagonists", "mineralocorticoid receptor antagonists", "spironolactone", "canrenone", "eplerenone", "all-cause mortality", "hospitalization", "hyperkalemia", and "gynecomasty" variously combined by means of the Boolean operators AND and OR. Titles and abstracts of all identified citations were reviewed independently by four authors (RDV, CC, DM, and AB) and any candidate study was selected for further screening of the full text. Possible discrepancies were resolved by consensus in 


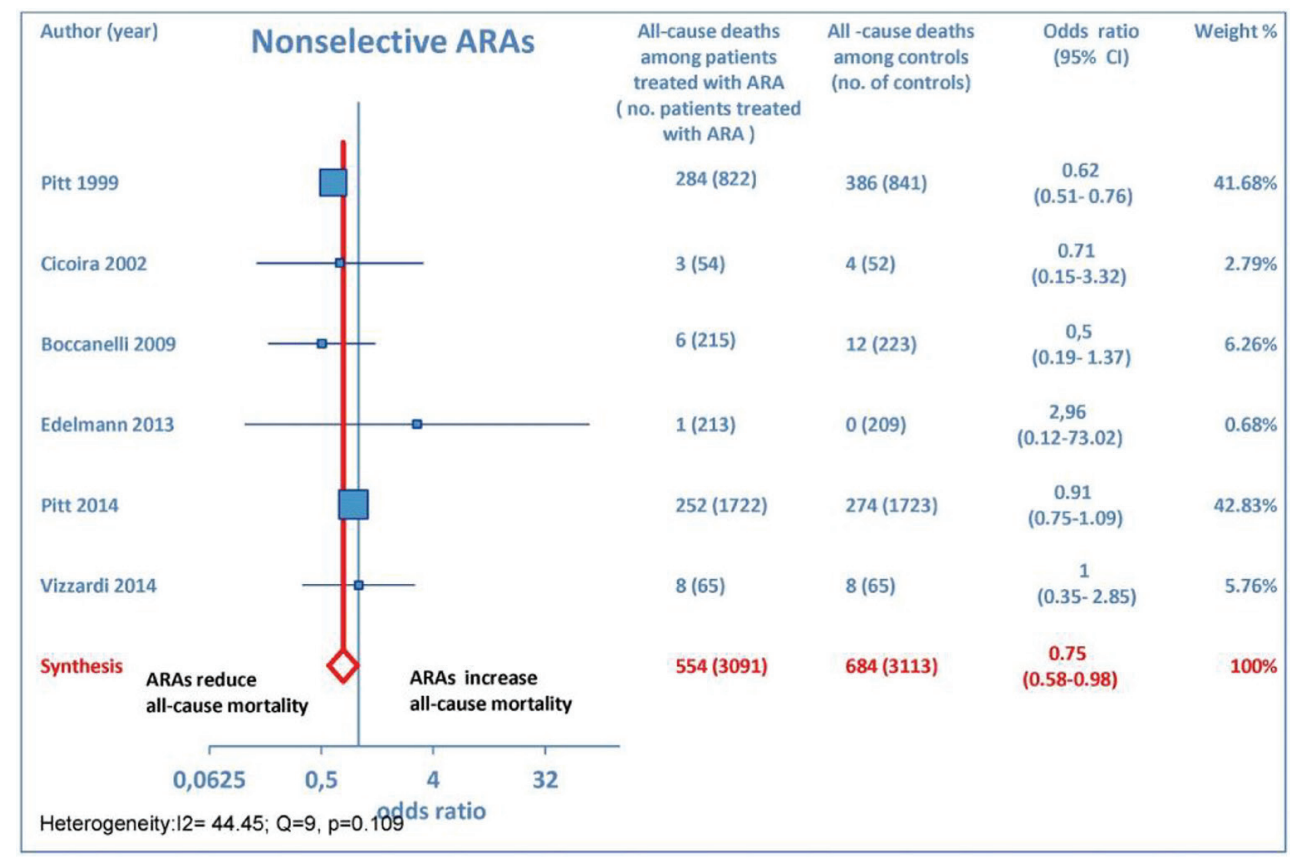

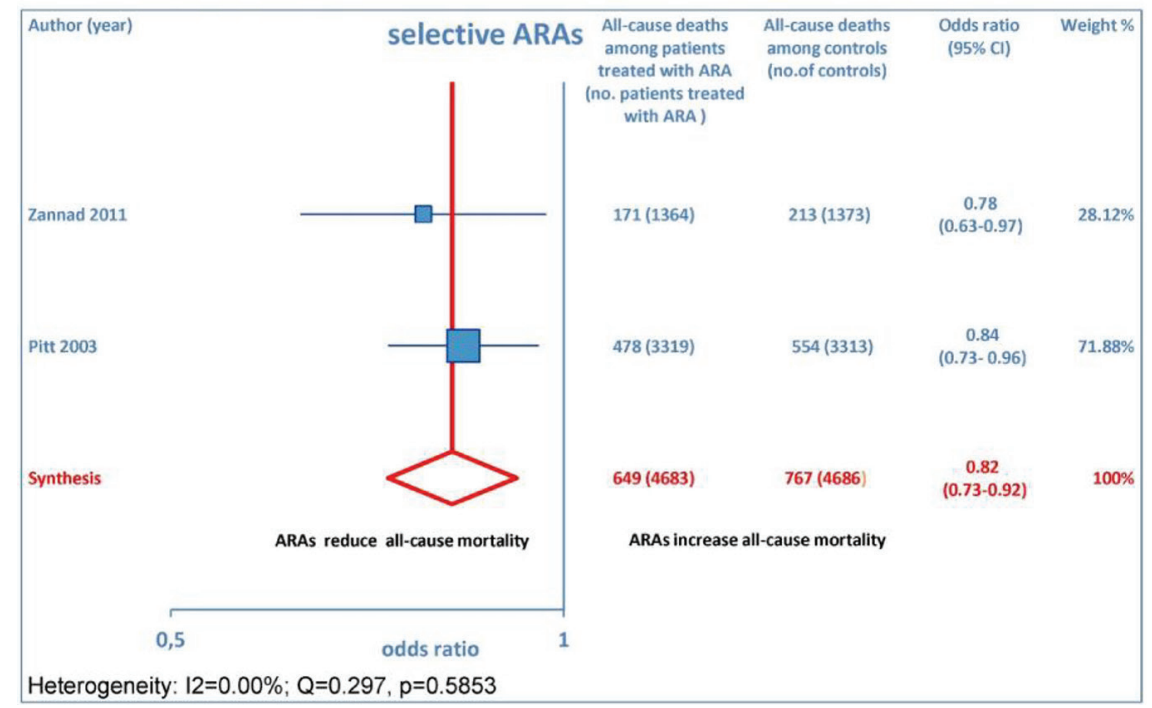

Figure 4. The forest plot of all-cause mortality has been subdivided according to ARA type (whether non-selective or selective).

consultation with a fifth author (NM). We extracted the following items from each study: population (type of heart failure and study size), intervention (ARA type), control (placebo, none, and other), and outcomes (all-cause mortality, cardiovascular mortality, hospitalizations, hyperkalemia, and gynecomastia).

\section{Statistical analysis}

Statistical analysis was performed using Review Manager 5.0.4 software (available from Cochrane Collaboration at http://www.cochrane.org) and Stata version 10 (Stata Corp LP, College station, TX, USA). For all of the considered efficacy or safety endpoints, the effect size, i.e., the effect of therapy with ARAs plus conventional therapy (ACE inhibitors, betablockers, and loop diuretics) versus conventional therapy alone was presented as an odds ratio (OR) with a 95\% confidence interval $(\mathrm{CI})$. We used a random effects model to pool results, and inverse variance as the weighting method. For quantifying statistical heterogeneity, the calculation of the $\mathrm{I}^{2}$ statistic $\left(\mathrm{I}^{2}\right)$ was also used, to represent the percentage of variability due to between-study variability. We rated $\mathrm{I}^{2}$ of less than $25 \%, 25-$ 


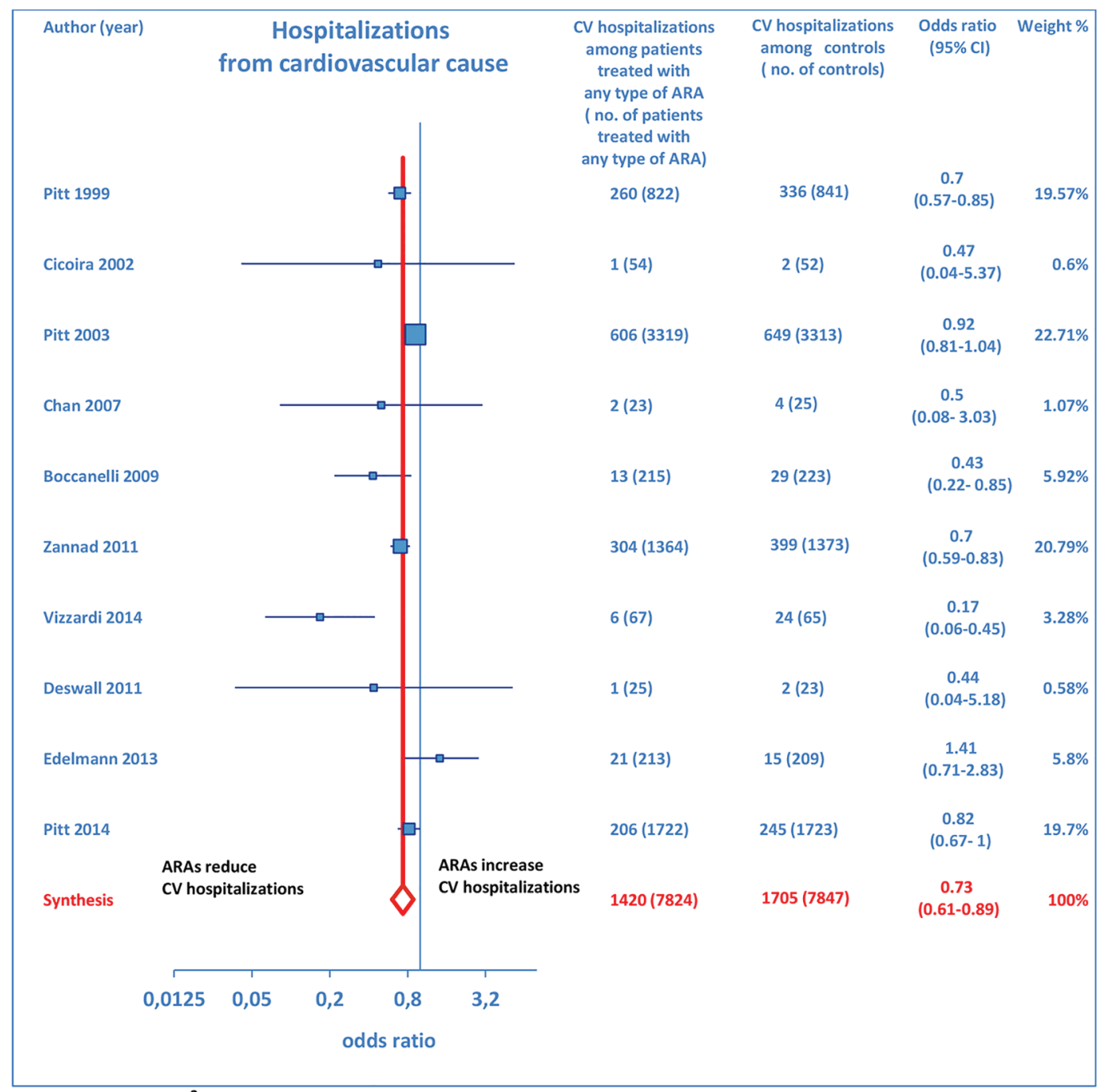

Heterogeneity: $I^{2}=64.20 \% ; Q=25.1421, p=0.0028$

Figure 5. Forest plot of hospitalizations from cardiovascular cause with ARA use in HF. Ten trials reported cardiovascular hospitalization rates with ARA use in HF patients compared to controls.

$50 \%$, and more than 50\% as low, moderate, and high amounts of heterogeneity, respectively. Publication bias was assessed using Begg's funnel plot. Results were regarded as statistically significant if $\mathrm{P}$ was less than 0.05 .

\section{Results}

One thousand seven hundred sixty citations were found; from these, in total, 36 studies were selected. Among them, 21 were eliminated after reading the article in full, due to incomplete or unavailable data, or because of ascertained inconsistency with our inclusion criteria. In particular, we excluded articles from the meta-analysis because of treatment in a non-heart failure setting (four studies), lack of relevant outcomes (13 studies), study duplication (three studies), and not an RCT design (one study; Fig. 1).

Overall, 15 RCTs comprising a total of 15,671 patients were eligible for inclusion in the meta-analysis. ARA use in patients with heart failure was associated with a significant reduction in adverse outcomes. Indeed, significantly reduced odds of all-cause death among CHF patients treated with ARAs compared to controls was found (OR: 0.79; 95\% CI: 0.73 0.87; $\mathrm{I}^{2}=30.67$ (moderate heterogeneity); Fig. 2). Subgroup analysis based on the HF type - whether HFREF or HFpEF - revealed a statistically significant benefit for patients with HFREF (OR: 0.77; 95\% CI: 0.69 - 0.84), whereas a protective effect against all-cause death was not attained by ARAs in the HFpEF subset (OR: 0.91; 95\% CI: 0.76 - 1.1; Fig. 3). Moreover, use of either a non-selective or selective ARA yielded a similarly significant reduction in all-cause mortality (Fig. 4).

Furthermore, as regards hospitalizations from cardiovascular cause (CV hosp), Figures 5 and 6 illustrate that significantly reduced odds of CV hosp were detected in the entire group of CHF patients under treatment with ARAs (OR: 0.73; 95\% CI: $0.61-0.89$ ) as well as among HFREF patients treated 

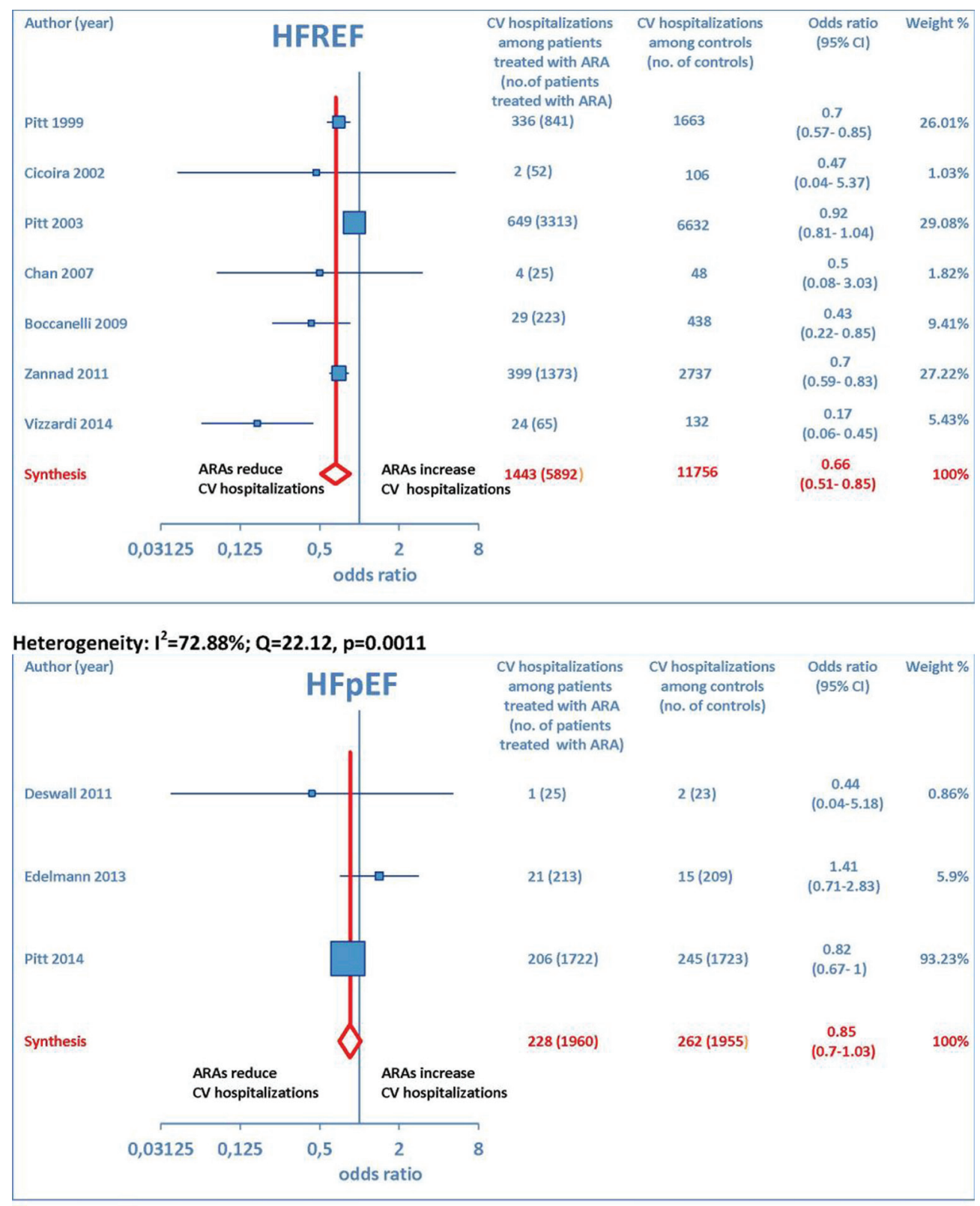

Heterogeneity: $I^{2}=19.57 \% ; Q=2.486, p=0.2884$

Figure 6. The forest plot of cardiovascular hospitalizations has been subdivided according to HF type.

with ARAs, compared to controls (OR: $0.66 ; 95 \%$ CI: 0.51 - 0.85). High heterogeneity was found as an accompanying feature $\left(\mathrm{I}^{2}=64.20 \%\right)$. Our a priori subgroup analysis partially explained the heterogeneity within this outcome, as a significant reduction in CV hosp was found in the HFREF (Fig. 6) and non-selective ARA subgroups (Fig. 7), whereas reduction in CV hosp in the selective ARA subset did not reach statistical significance (Fig. 7). Hyperkalemia was significantly more common with ARA use (Fig. 8).

In addition, subgroup analysis by ARA type documented that both non-selective and selective ARAs were similarly associated with increased odds of episodes of hyperkalemia compared to controls (Fig. 9).

ARA use was shown to be associated with the occurrence of gynecomastia (Fig. 10). In particular, selective ARAs proved not to produce significant amounts of gynecomastia compared to controls (OR: 0.74; 95\% CI: 0.43 - 1.27), while non-selective ARAs did (OR: 8.22; 95\% CI: 4.9 - 13.81; Fig. $11)$.

\section{Discussion}

In this meta-analysis, we tried to assess the impact of ARAs on 


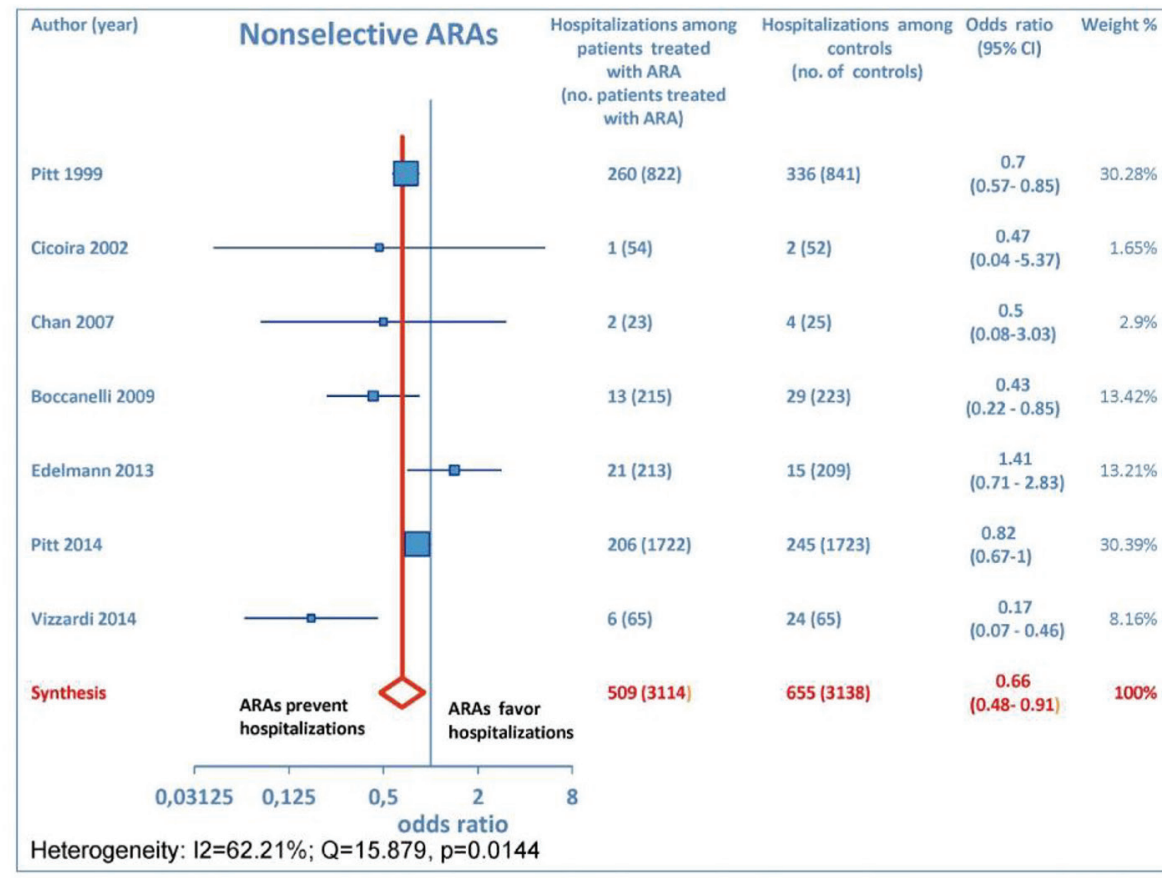

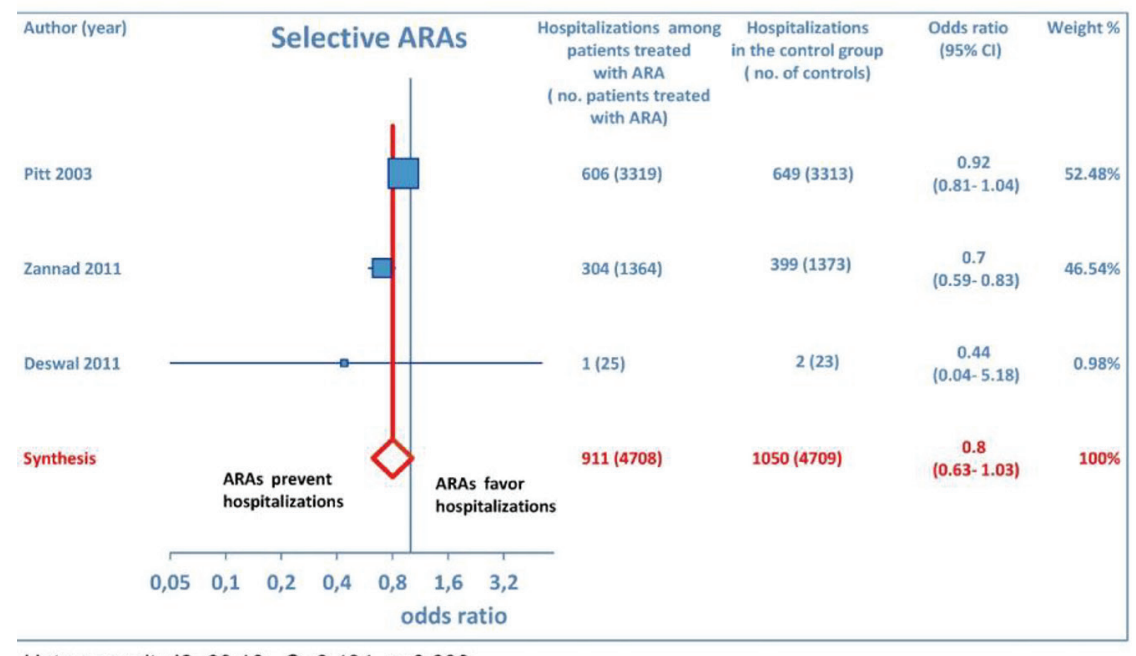

Heterogeneity: $I 2=69.16 ; Q=6.484, p=0.039$

Figure 7. The forest plot of cardiovascular hospitalizations has been subdivided according to ARA type (whether non-selective or selective).

several efficacy and safety endpoints by maintaining distinct the outcomes detected in HFREF from those associated with HFpEF. Furthermore, for both efficacy and safety outcomes in the setting of $\mathrm{CHF}$, separate meta-analyses were performed for RCTs centered on non-selective ARAs and for those which had investigated selective ARAs.

ARA use in patients with heart failure was associated with a significant reduction in all-cause death (OR: $0.79 ; 95 \% \mathrm{CI}$ : 0.73 - 0.87; Fig. 2) and CV hosp (OR: 0.73; 95\% CI: 0.61 - 0.89; Fig. 5). However, judging by our subgroup analysis, the favorable effects of ARAs on the efficacy endpoints were limited to HFREF; conversely, ARA-related reductions concerning all-cause mortality and CV hosp in HFpEF patients did not reach statistical significance (Figs. 3 and 6, respectively). Both selective and non-selective ARAs increased the risk of hyperkalemia in a similar manner. Instead, gynecomastia was limited to non-selective ARAs; indeed, non-selective ARAs exhibited an OR of 8.22 (95\% CI: 4.9 - 13.81), as opposed to selective ARAs which showed a non-significant OR of 0.74 (95\% CI: 0.43 - 1.27; Fig. 11). 


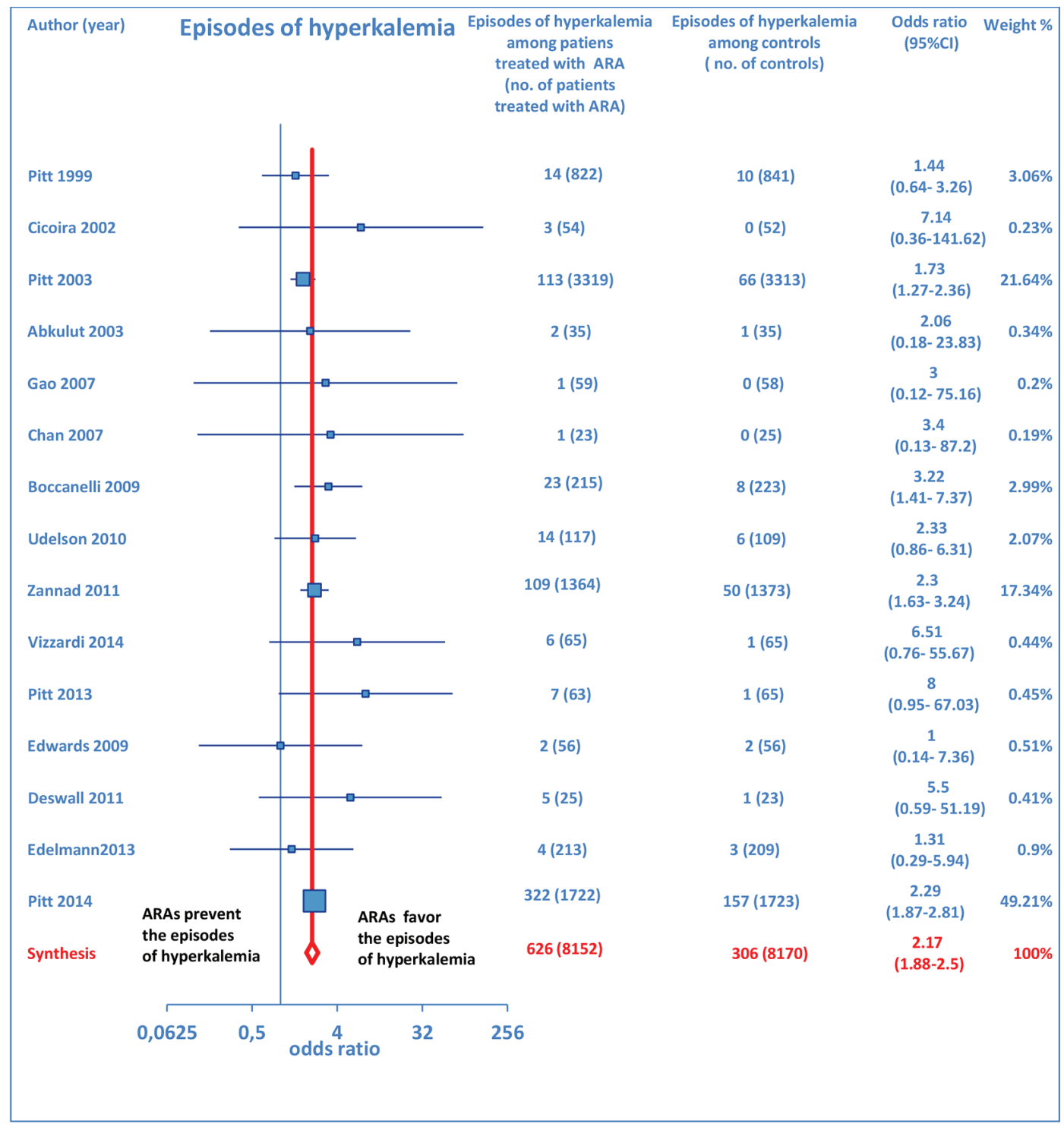

Heterogeneity: $I^{2}=0.00 \% ; Q=9.1439, p=0.8217$

Figure 8. Forest plot of hyperkalemia with ARA use in HF. Fifteen trials reported hyperkalemia rates with ARA use in HF patients compared to controls.

Overall, the evidence supporting ARA use in HFREF was based on a large number of trials with significant effect sizes for reducing adverse cardiac events. In contrast, the evidence for ARA use in HFpEF was based on a relatively small number of trials, only one of which had a significant reduction in $\mathrm{CV}$ hosp but no significant effect on all-cause mortality [15]. Finally, our conclusions support ARA use in HFREF, in keeping with current American Heart Association guidelines, which recommend ARAs for patients with HFREF and NYHA class II-IV symptoms or following acute myocardial infarction complicated by heart failure and LVEF $\leq 40 \%$ [16]

On the whole, no significant effects of ARAs on efficacy outcomes (all-cause mortality and CV hosp) concerning HF-
$\mathrm{pEF}$ were demonstrated by the present meta-analysis. Furthermore, ARAs were associated with a significantly increased risk of some harmful side effects, such as hyperkalemia and gynecomastia. In this meta-analysis, selective ARAs proved to offer a slight benefit in terms of no significant gynecomastia while having equivalent reductions in adverse cardiac outcomes with respect to non-selective ARAs. Based on our meta-analysis, we would advise continued usage of ARAs in HFREF, where there is a significant reduction in adverse cardiac outcomes, such as all-cause mortality and CV hosp. Instead, based on the present meta-analysis, in our opinion, the choice of administering an ARA to patients with HFpEF is rather questionable, because in this type of CHF, the ARAs-related reductions in adverse cardiovascular outcomes did not reach statistical sig- 


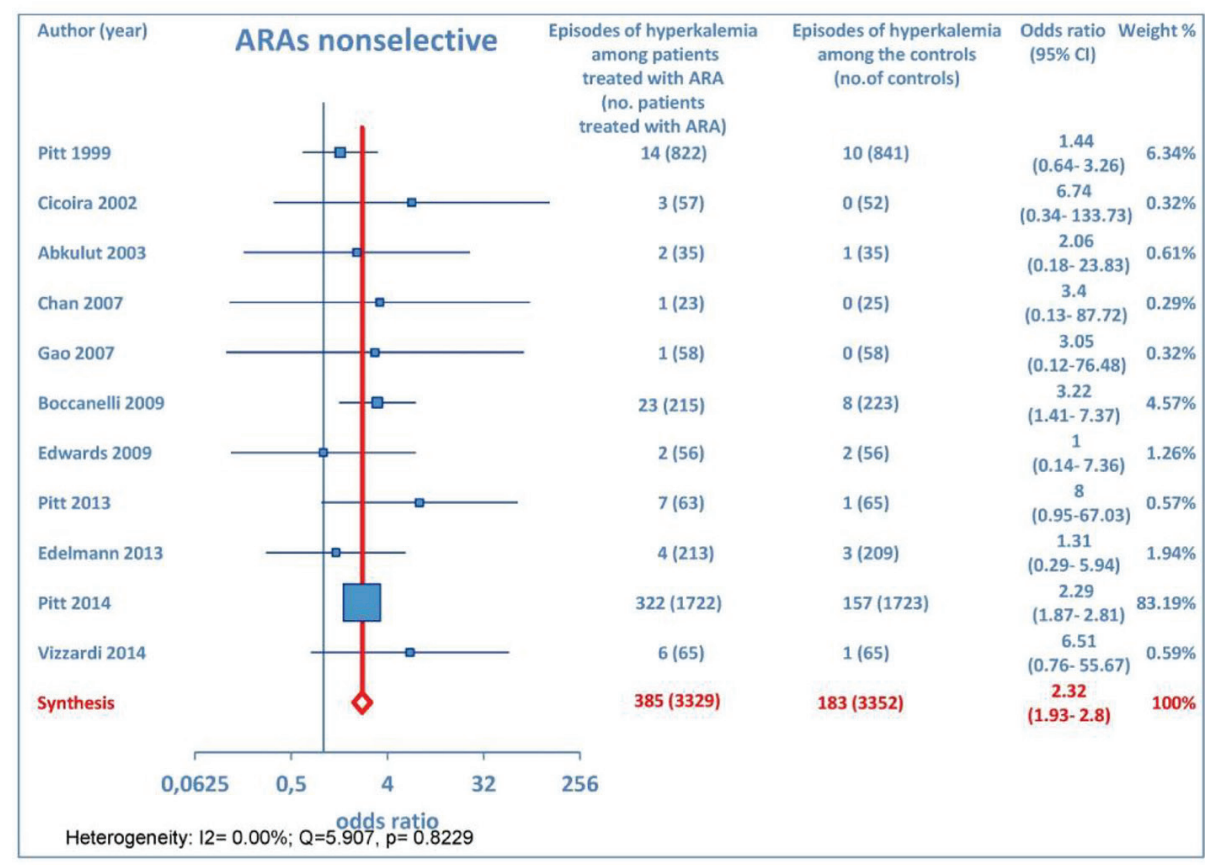

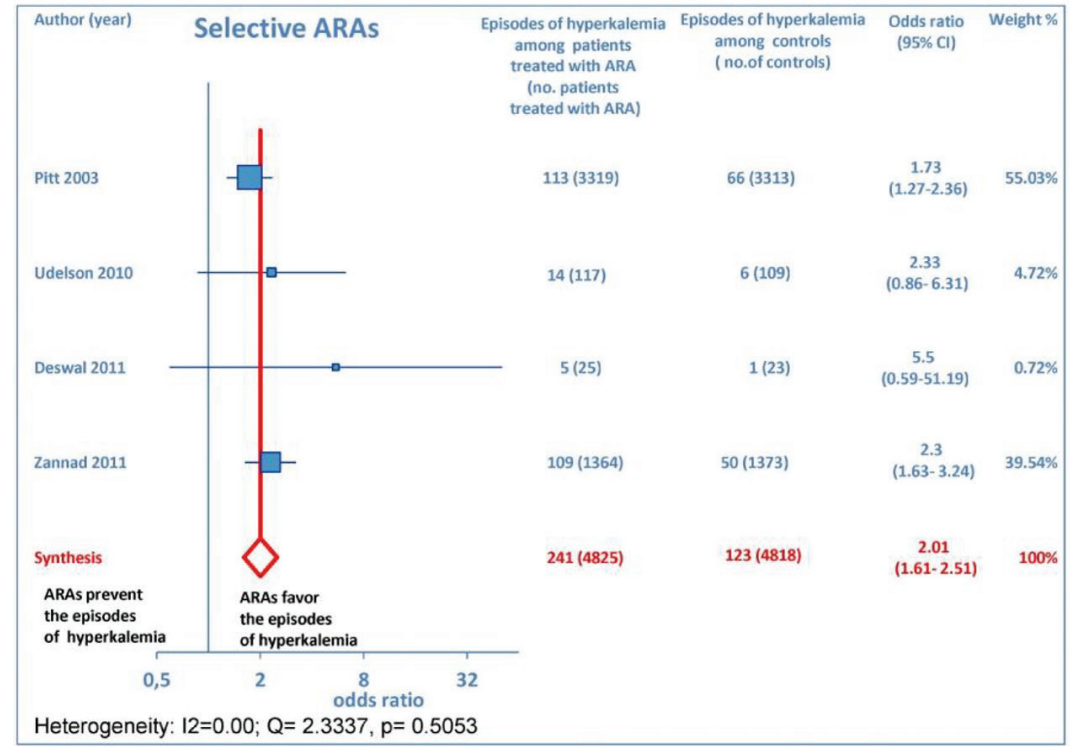

Figure 9. The forest plot of hyperkalemia has been subdivided according to ARA type (whether non-selective or selective).

nificance.

\section{Hyperkalemia during ARA therapy: a challenging issue}

Our meta-analysis was not specifically aimed at evaluating the approach with ARAs in the setting of cardio-renal syndromes, i.e., the conditions characterized by the coexistence of cardiac and renal failure [17-19]. However, some points would deserve more thorough awareness in the medical community. In particular, current rough epidemiological calculations [20] indicate that about a third of the general population aged $>70$ years is affected by a reduction in the estimated glomerular filtration rate (eGFR) of $<60 \mathrm{~mL} / \mathrm{min} / 1.73 \mathrm{~m}^{2}$ and therefore has a chronic kidney disease (CKD) by definition [21]. However, a stable low eGFR in the elderly, when it is physiologically sufficient to satisfy the homeostatic demands, is not a disease per se and seldom progresses up to an advanced renal failure [21]. Moreover, it would be appropriate to underline that a large proportion of patients with CHF and concomitant renal insufficiency may have a simple overlapping of the two dysfunctions, cardiac and renal, with no possibility of documenting a 


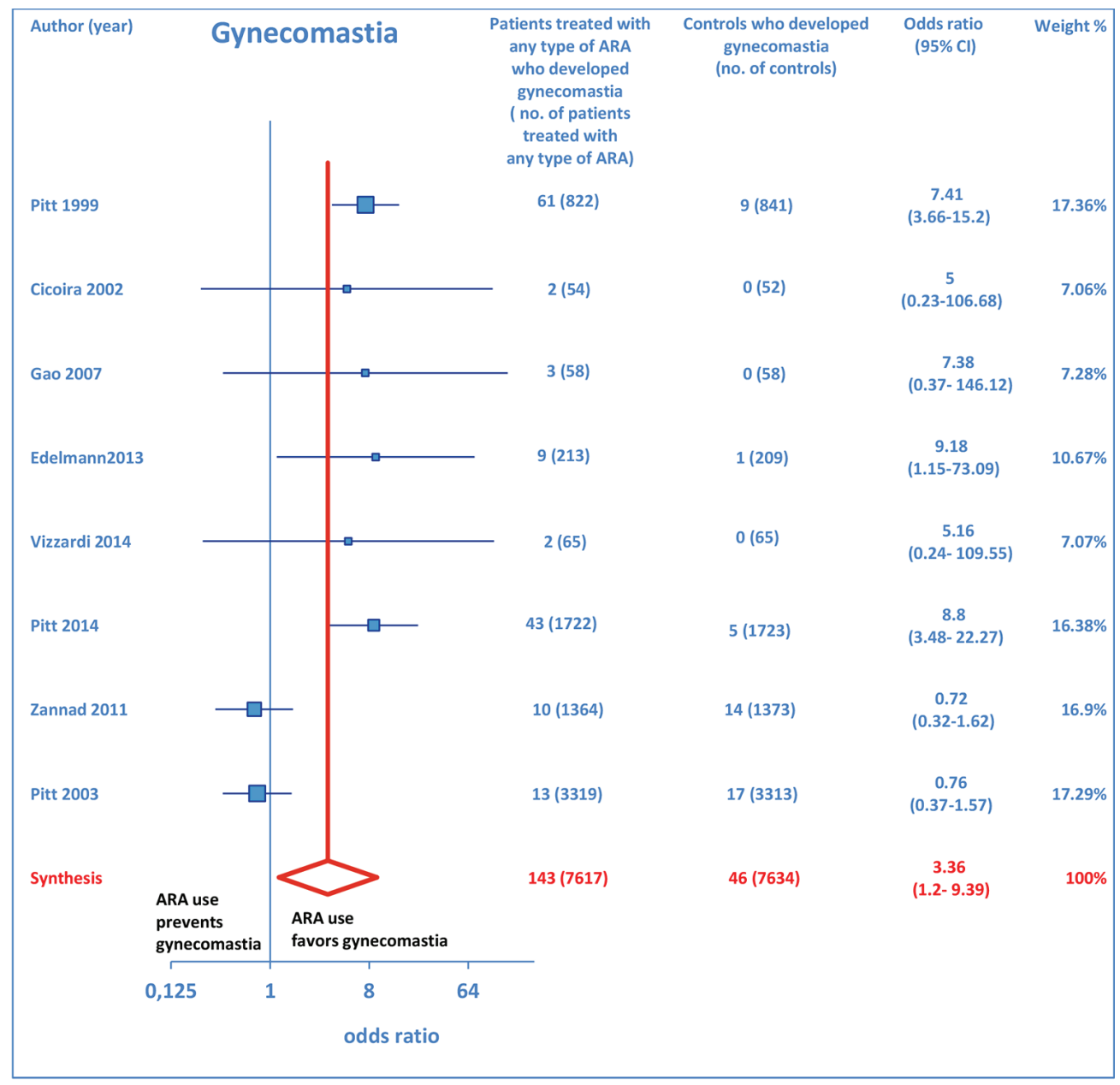

Heterogeneity: $I^{2}=81.54 \% ; Q=37.9273, p=3.12801 E-06$

Figure 10. Forest plot of gynecomastia with ARA use in HF. Eight trials reported gynecomastia rates with ARA use in HF patients compared to controls.

cardiorenal syndrome type 2 .

Notably, the most important RCTs incorporated in our meta-analysis did not exclude CHF patients with concomitant mild to moderate renal insufficiency. For instance, in the EMPHASIS-HF study by Zannad et al [12] as well as in the TOPCAT study by Pitt et al [15], only severe renal dysfunction was excluded, as opposed to mild-to-moderate renal insufficiency. Indeed, in the Methods of both studies, it is affirmed that severe renal dysfunction (an eGFR of $<30 \mathrm{~mL} / \mathrm{min} / 1.73 \mathrm{~m}^{2}$ of body surface area or a serum creatinine $\geq 2.5 \mathrm{mg} / \mathrm{dL}$ ) was an exclusion criterion. Instead, the recruitment of CHF patients with a lesser degree of renal dysfunction was not banned. So it is reasonable to affirm that the populations of these studies did not consist of "enclaves" isolated from the real world. In fact in these patients, the comorbidity "renal failure" (due to atherosclerotic ischemic renal disease, hypertensive or diabetic nephropathy, etc.) was adequately represented.

Nevertheless, an effective and safe dosing modulation concerning evidence-based therapies for CHF, i.e., beta-blockers, ACE inhibitors, ARBs and ARAs, remains a challenging problem, which requires wise clinical choices, in addition to the adherence to few inclusion and exclusion criteria. In particular, with regard to the conditions in which ARA treatment has to be banned, an important one is avoiding ARA therapy in CHF patients with advanced renal insufficiency (GFR $<30$ $\mathrm{mL} / \mathrm{min} / 1.73 \mathrm{~m}^{2}$ ), in whom even the use of reduced doses of ACE inhibitor, e.g., enalapril at a dose of $2.5-5 \mathrm{mg} /$ day, cannot be regarded as a sufficient precaution to avert the risk of hyperkalemia. Thus, in advanced CKD as well as in any case of acute kidney injury superimposed on a condition of chronic cardiac decompensation, ARA use should be mandatorily avoided in order to prevent the impending risk of dangerous episodes of hyperkalemia.

However, in CHF patients with only mild or moderate CKD (GFR calculated between 30 and $60 \mathrm{~mL} / \mathrm{min} / 1.73 \mathrm{~m}^{2}$ by using the modified diet in renal disease (MDRD) equation [22]) the simultaneous use of various types of drugs blocking the RAAS - in particular, ACE inhibitors plus ARAs or, alternatively, ARBs plus ARAs - should not be impeded but rather it should be adequately modulated and carefully monitored [17]. 

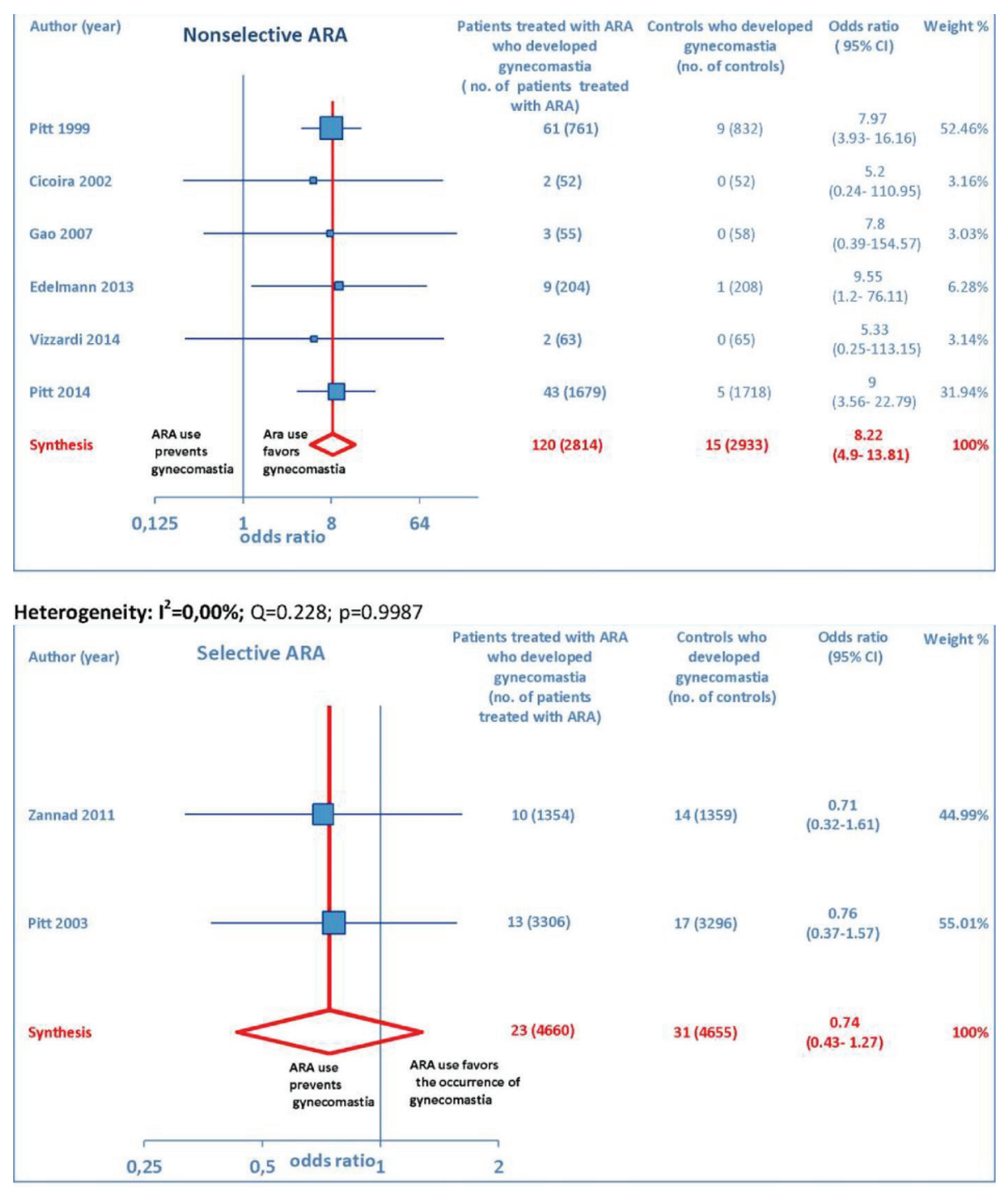

Heterogeneity: $I^{2}=0.00 \% ; Q=0.0129 ; p=0.9095$

Figure 11. The forest plot of gynecomastia has been subdivided according to ARA type (whether non-selective or selective).

Proper patient selection, including patients with diminished LVEF and excluding ones with moderate CKD (creatinine level $\geq 2.5 \mathrm{mg} / \mathrm{dL}$ or serum $\mathrm{K}^{+}>5 \mathrm{mEq} / \mathrm{L}$ ), would help minimize potential life-threatening hyperkalemia [23].

\section{Conclusions}

In addition to already existing supportive data, our metaanalysis provides further evidence that ARAs should be systematically used in patients with HFREF, where they improve some hard clinical endpoints, such as all-cause mortality and hospitalizations from cardiac cause. Conversely, based on the present meta-analysis, ARA usage in HFpEF patients is questionable since in this CHF setting, no significant improvement in clinical endpoints has been demonstrated so far, in the face of the well-known risks of hyperkalemia and/or gynecomastia that chronic ARA therapy entails. Furthermore, new selective ARAs are not burdened by significant risk of gynecomastia, while they are similar to non-selective ARAs with regard to the efficacy profile as well as to the risk of eliciting hyperkalemia.

\section{References}

1. Masoumi A, Ortiz F, Radhakrishnan J, Schrier RW, Colombo PC. Mineralocorticoid receptor antagonists as diuretics: Can congestive heart failure learn from liver failure? Heart Fail Rev. 2015;20(3):283-290.

2. Schrier RW. Body fluid volume regulation in health and disease: a unifying hypothesis. Ann Intern Med. 1990;113(2):155-159.

3. Schrier RW. Water and sodium retention in edematous 
disorders: role of vasopressin and aldosterone. Am J Med. 2006;119(7 Suppl 1):S47-53.

4. Schrier RW. Decreased effective blood volume in edematous disorders: what does this mean? J Am Soc Nephrol. 2007;18(7):2028-2031.

5. Schrier RW, Abraham WT. Hormones and hemodynamics in heart failure. N Engl J Med. 1999;341(8):577-585.

6. Gheorghiade M, Filippatos G, De Luca L, Burnett J. Congestion in acute heart failure syndromes: an essential target of evaluation and treatment. Am J Med. 2006;119(12 Suppl 1):S3-S10.

7. McKelvie RS, Yusuf S, Pericak D, Avezum A, Burns RJ, Probstfield J, Tsuyuki RT, et al. Comparison of candesartan, enalapril, and their combination in congestive heart failure: randomized evaluation of strategies for left ventricular dysfunction (RESOLVD) pilot study. The RESOLVD Pilot Study Investigators. Circulation. 1999;100(10):1056-1064.

8. Struthers A, Krum H, Williams GH. A comparison of the aldosterone-blocking agents eplerenone and spironolactone. Clin Cardiol. 2008;31(4):153-158.

9. Pitt B. Aldosterone blockade in patients with chronic heart failure. Cardiol Clin. 2008;26(1):15-21, v.

10. Pitt B, Zannad F, Remme WJ, Cody R, Castaigne A, Perez A, Palensky J, et al. The effect of spironolactone on morbidity and mortality in patients with severe heart failure. Randomized Aldactone Evaluation Study Investigators. N Engl J Med. 1999;341(10):709-717.

11. Pitt B, Remme W, Zannad F, Neaton J, Martinez F, Roniker B, Bittman R, et al. Eplerenone, a selective aldosterone blocker, in patients with left ventricular dysfunction after myocardial infarction. $N$ Engl J Med. 2003;348(14):1309-1321.

12. Zannad F, McMurray JJ, Krum H, van Veldhuisen DJ, Swedberg K, Shi H, Vincent J, et al. Eplerenone in patients with systolic heart failure and mild symptoms. N Engl J Med. 2011;364(1):11-21.

13. de Gasparo M, Joss U, Ramjoue HP, Whitebread SE, Haenni H, Schenkel L, Kraehenbuehl C, et al. Three new epoxy-spirolactone derivatives: characterization in vivo and in vitro. J Pharmacol Exp Ther. 1987;240(2):650656.

14. Moher D, Liberati A, Tetzlaff J, Altman DG. Preferred reporting items for systematic reviews and meta-analyses: the PRISMA statement. BMJ. 2009;339:b2535.

15. Pitt B, Pfeffer MA, Assmann SF, Boineau R, Anand IS, Claggett B, Clausell N, et al. Spironolactone for heart failure with preserved ejection fraction. N Engl J Med. 2014;370(15):1383-1392.

16. Yancy CW, Jessup M, Bozkurt B, Butler J, Casey DE, Jr., Drazner MH, Fonarow GC, et al. 2013 ACCF/AHA guideline for the management of heart failure: executive summary: a report of the American College of Cardiology Foundation/American Heart Association Task Force on practice guidelines. Circulation. 2013;128(16):18101852.

17. Ronco C, Haapio M, House AA, Anavekar N, Bellomo R. Cardiorenal syndrome. J Am Coll Cardiol. 2008;52(19):1527-1539.

18. Boerrigter G, Hocher B, Lapp H. Changes in renal function in congestive heart failure. Curr Heart Fail Rep. 2013;10(4):285-295.

19. De Vecchis R, Baldi C. Cardiorenal syndrome type 2: from diagnosis to optimal management. Ther Clin Risk Manag. 2014;10:949-961.

20. K/DOQI clinical practice guidelines for chronic kidney disease: evaluation, classification, and stratification. Am J Kidney Dis. 2002;39(2 Suppl 1):S1-266.

21. Winearls CG, Glassock RJ. Classification of chronic kidney disease in the elderly: pitfalls and errors. Nephron Clin Pract. 2011;119(Suppl 1):c2-4.

22. Levey AS, Bosch JP, Lewis JB, Greene T, Rogers N, Roth D. A more accurate method to estimate glomerular filtration rate from serum creatinine: a new prediction equation. Modification of Diet in Renal Disease Study Group. Ann Intern Med. 1999;130(6):461-470.

23. Ko DT, Juurlink DN, Mamdani MM, You JJ, Wang JT, Donovan LR, Tu JV. Appropriateness of spironolactone prescribing in heart failure patients: a population-based study. J Card Fail. 2006;12(3):205-210. 